\title{
Comparison of trimethylgallium and triethylgallium as "Ga" source materials for the growth of ultrathin GaN films on Si (100) substrates via hollow-cathode plasma-assisted atomic layer deposition
}

\author{
Mustafa Alevlia) \\ Department of Physics, Marmara University, Göztepe Kadıköy, 34722 Istanbul, Turkey \\ Ali Haider, Seda Kizir, Shahid A. Leghari, and Necmi Biyiklib) \\ Institute of Materials Science and Nanotechnology, Bilkent University, Bilkent, 06800 Ankara, Turkey \\ and National Nanotechnology Research Center (UNAM), Bilkent University, Bilkent, 06800 Ankara, Turkey
}

(Received 5 September 2015; accepted 30 November 2015; published 21 December 2015)

\begin{abstract}
GaN films grown by hollow cathode plasma-assisted atomic layer deposition using trimethylgallium (TMG) and triethylgallium (TEG) as gallium precursors are compared. Optimized and saturated TMG/TEG pulse widths were used in order to study the effect of group-III precursors. The films were characterized by grazing incidence $\mathrm{x}$-ray diffraction, atomic force microscopy, $\mathrm{x}$-ray photoelectron spectroscopy, and spectroscopic ellipsometry. Refractive index follows the same trend of crystalline quality, mean grain, and crystallite sizes. GaN layers grown using TMG precursor exhibited improved structural and optical properties when compared to GaN films grown with TEG precursor. @ 2015 American Vacuum Society. [http://dx.doi.org/10.1116/1.4937725]
\end{abstract}

\section{INTRODUCTION}

The enormous potential of gallium nitride $(\mathrm{GaN})$ based electronics and optoelectronics is still being limited by the absence of commercially available native substrates with large diameters at reasonable cost levels. Instead, GaN device layers are most widely grown on non-native substrate materials like sapphire and $\mathrm{SiC}$. Compared to Si substrates, both these materials perform short at the available wafer size and materials cost. Therefore, researchers have paid more attention to the growth of GaN films on various unconventional oxide and metallic substrates. Besides, many researchers have devoted significant effort in the growth of $\mathrm{GaN}$ films on Si substrates, which is an ongoing active research area with promising results and even commercial success. ${ }^{1-3}$ The integration of GaN-based RF-microwave and optoelectronic devices with highly advanced CMOS electronic backbone might significantly increase the functionality, performance, and application spectrum of III-nitride devices. ${ }^{4}$ Most of the GaN-on-Si research has been focused on the development of GaN layers on Si (111) substrates due to the threefold surface symmetry and lowest amount of lattice mismatch of this particular plane. GaN layers grown on $\mathrm{Si}$ (111) using various buffer layers showed low impurity concentrations and decent electrical properties; however, $\mathrm{Si}$ (100) is still known as the standard substrate for mainstream Si-based CMOS technology. ${ }^{3}$ Therefore, the development of high-quality $\mathrm{GaN}$ on $\mathrm{Si}(100)$ is necessary for the integration of GaN HEMTs with advanced Si electronics and III-nitride light emitting diodes with enhanced light output. On the other hand, the mismatch in thermal expansion coefficients between $\mathrm{Si}(100)$ and $\mathrm{GaN}$ causes large tensile stress in asgrown films during cool-down period from the typical high growth temperatures $\left(\sim 1000^{\circ} \mathrm{C}\right)$ and results in cracks which

\footnotetext{
${ }^{a)}$ Electronic mail: mustafaalevli@marmara.edu.tr

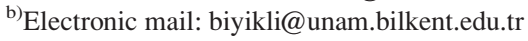

reduces the performance of GaN-based devices. ${ }^{5}$ When epitaxial growth of $\mathrm{GaN}$ directly takes place on $\mathrm{Si}$ at high growth temperatures, surface atoms will easily react with nitrogen, which forms $\mathrm{SiN}_{\mathrm{x}}$ interfacial layer and considerably hinders the successive GaN epitaxy. In order to overcome these problems, metal-organic vapor deposition (MOCVD) and molecular beam epitaxy runs at reduced substrate temperatures have been carried out. However, those GaN films show poor crystal quality due to the substantially low kinetic energy which is required for the thermodynamically favorable migration of precursors over the substrate surface. ${ }^{5}$ In order to overcome the limitations due to high substrate temperatures and the need for complex buffer layers, ${ }^{6}$ among alternative approaches for low-temperature growth of $\mathrm{GaN}$ include physical vapor deposition (PVD) techniques (sputtering and pulsed laser deposition) as well as a more-recently developed chemical vapor deposition technique: plasma-assisted atomic layer deposition (PA-ALD). ${ }^{7-9}$ When compared with the alternative lowtemperature PVD techniques, PA-ALD stands out in terms of large-area uniformity, submonolayer precision thickness control, and ultimate conformality. Moreover, PA-ALD growth of GaN films might be optimized in a self-limiting growth regime, where precursors do not need high kinetic energy for migration, making PA-ALD a powerful method over other low-temperature growth techniques. These features make PAALD method suitable for deposition on temperature sensitive device layers and flexible polymer substrates as well. ${ }^{10}$

PA-ALD might be a potential alternative pathway for low-temperature deposition of $\mathrm{GaN}$ due its unique characteristics including uniformity, conformality, good reproducibility, precise thickness, and stoichiometry control of the films. Partially reflecting this emerging potential, an increasing number of notable contributions appeared on the lowtemperature $\left(<300^{\circ} \mathrm{C}\right)$ growth of $\mathrm{GaN}$ films using PA-ALD with improved crystalline quality and electrical properties recently. ${ }^{6,11,12}$ In those reports, PA-ALD of GaN using 
trimethylgallium $(\mathrm{TMG})^{11} /$ triethylgallium (TEG), ${ }^{6,12}$ and $\mathrm{N}_{2} / \mathrm{H}_{2}$ plasma as the precursors were reported. However, no study exists yet which compares the influence of these gallium precursors on the optical and structural properties of PA-ALD grown GaN films.

In this work, we report a comparative study on GaN thin films grown by a specific PA-ALD technique, namely, hollow-cathode plasma-assisted atomic layer deposition, using TMG and TEG as group-III precursors. The GaN films analyzed were deposited using $\mathrm{N}_{2} / \mathrm{H}_{2}$ plasma as the nitrogen source at $200^{\circ} \mathrm{C}$ substrate temperature.

\section{EXPERIMENT}

GaN samples were grown on $\mathrm{Si}(100)$ substrates at $200^{\circ} \mathrm{C}$ using a modified Fiji F200-LL ALD reactor (Ultratech/ Cambridge Nanotech, Inc.) in which quartz-based ICP source was replaced with a stainless steel hollow-cathode plasma source (Meaglow, Inc.). For comparison purpose, the films were grown with identical parameters except for Ga precursor pulse durations due to the vapor pressure difference of precursors. Ga precursor exposure doses were adjusted in order to achieve the self-limiting growth scheme, which is an evidence of ideal atomic layer deposition (ALD) growth mechanism. ${ }^{11}$ Each ALD cycle consisted of four steps: $0.03 \mathrm{~s}$ TMG $/ 0.5 \mathrm{~s}$ TEG, $10 \mathrm{~s}$ Ar purge, $40 \mathrm{~s} 50+50$ sccm $300 \mathrm{~W} \mathrm{~N}_{2} / \mathrm{H}_{2}$ plasma, $10 \mathrm{~s}$ Ar purge, repeated for 1750 cycles in order to deposit TEG-grown GaN films and 2727 cycles in order to deposit TMG-grown GaN films.

The film thicknesses and optical constants were measured by spectroscopic ellipsometer (V-VASE, J. A. Woollam Co., Inc.) in the wavelength range of $300-1000 \mathrm{~nm}$ at various incidence angles. Crystalline phases of the GaN films were studied with grazing-incidence $\mathrm{x}$-ray diffraction (GIXRD) measurements (PANalytical X'Pert PRO MRD diffractometer) in $\Theta-2 \Theta$ mode with $\mathrm{Cu} \mathrm{K} \alpha$ radiation. Surface morphology was investigated by using atomic force microscopy (AFM, Park systems Corporation, XE-100). Thermo Scientific K-Alpha spectrometer with a monochromatized Al $\mathrm{K} \alpha \mathrm{X}$-ray source was used for the X-ray photoelectron spectroscopy (XPS) studies. Fourier transform infrared reflectance (FTIR) spectra were taken at room temperature and at normal incidence of IR radiation in the range of $450-700 \mathrm{~cm}^{-1}(22.22-14.28 \mu \mathrm{m})$ having a spectral resolution of $2 \mathrm{~cm}^{-1}$ by using a FTIR spectrometer (Bruker Vertex 70) with a mirror optics microscope (hyperion microscope) and a liquid nitrogen cooled MCT detector. All spectra were normalized to the reflection of a gold mirror.

\section{RESULTS AND DISCUSSION}

In order to establish the optimized deposition parameters for the growth of $\mathrm{GaN}$ under self-limiting growth conditions, the variation of growth rate per cycle (GPC) was studied for both Ga precursors as a function of exposure time, as shown in Fig. 1. The growth rate was defined in terms of the film thickness divided by the total applied ALD cycles. The film thicknesses were calculated using Cauchy dispersion function. The growth rate saturated for 0.03 and $0.5 \mathrm{~s}$ of TMG

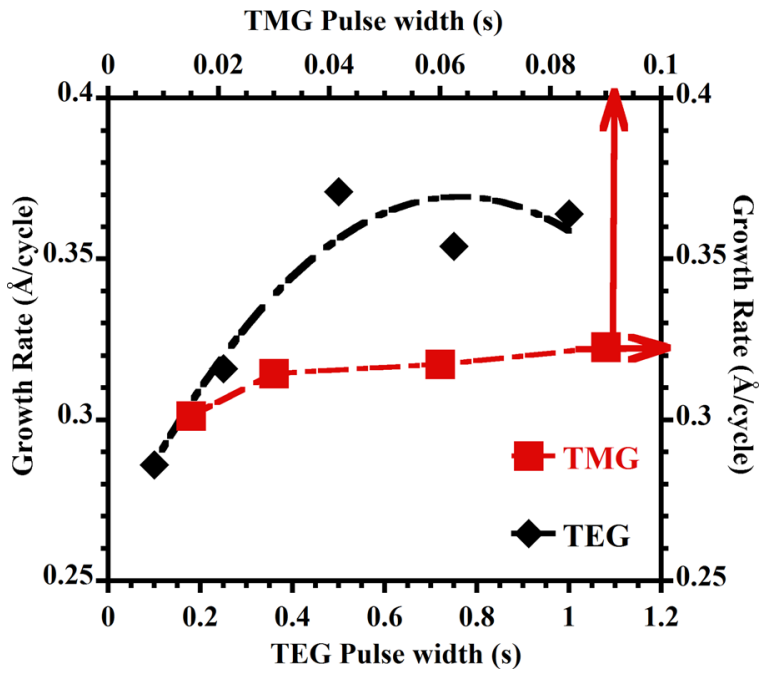

Fig. 1. (Color online) TEG and TMG saturation curves at $200^{\circ} \mathrm{C}$. The $\mathrm{N}_{2} / \mathrm{H}_{2}$ flow rate and flow duration were kept constant at $50+50 \mathrm{sccm}$ and $40 \mathrm{~s}$, respectively.

and TEG doses, respectively. Both saturation curves are indicators of self-limited growth. The difference in GPC values might be attributed to the different physical and chemical properties (sticking coefficient, nucleation behavior, steric hindrance effect, etc.) of group-III precursor molecules. ${ }^{13}$

In order to identify the crystalline nature of the GaN films, GIXRD measurements were performed. As revealed by their GIXRD patterns, GaN films showed single-phase polycrystalline character with hexagonal wurtzite crystallite structure due to the existence of multiple GaN diffraction planes. The same reflection peaks were observed for both samples and were referred to h-GaN (002) and h-GaN (103). No other phase mixing was detected (i.e., cubic). The use of different metal alkyl precursors affected neither peak positions nor the diffraction planes. It can be seen that the (002) diffraction peak intensity slightly increases with the use of TMG precursor [Fig. 2(a)]. The (002) peak at $34.62^{\circ}$ for TMG-grown GaN and $34.63^{\circ}$ for TEG-grown $\mathrm{GaN}$ correspond to the approximately same lattice parameters $a=3.184 \AA, c=5.176 \AA$, which is consistent with the reported values for bulk GaN. ${ }^{1}$ Crystallite size values were calculated from the (002) reflections using line profile analysis and found to be 14.25 (TMG$\mathrm{GaN}$ ) and $12.98 \mathrm{~nm}$ (TEG-GaN). Assuming the line broadening [FWHM of (002) diffraction peak], the lattice strain increases with the use of TEG precursor. Both GaN films grown at low temperatures with TMG and TEG precursors exhibited polycrystalline character. The surface roughness and grain size of the GaN films were obtained by analyzing the AFM images [Figs. 2(b) and 2(c)]. Rms surface roughness values measured from a $1 \times 1 \mu \mathrm{m}^{2}$ scan area of the samples grown with TMG and TEG were recorded as 1.51 and $0.37 \mathrm{~nm}$, respectively. The mean grain sizes were obtained from the analysis of AFM images as 21.2 and $5.6 \mathrm{~nm}$ for TMG- and TEG-grown GaN samples, respectively. The surface of TEG-grown film is mostly 2D with occasional bumps whereas the entire surface of the TMG-grown sample is covered with larger hillocks. Those results indicate that the use of 

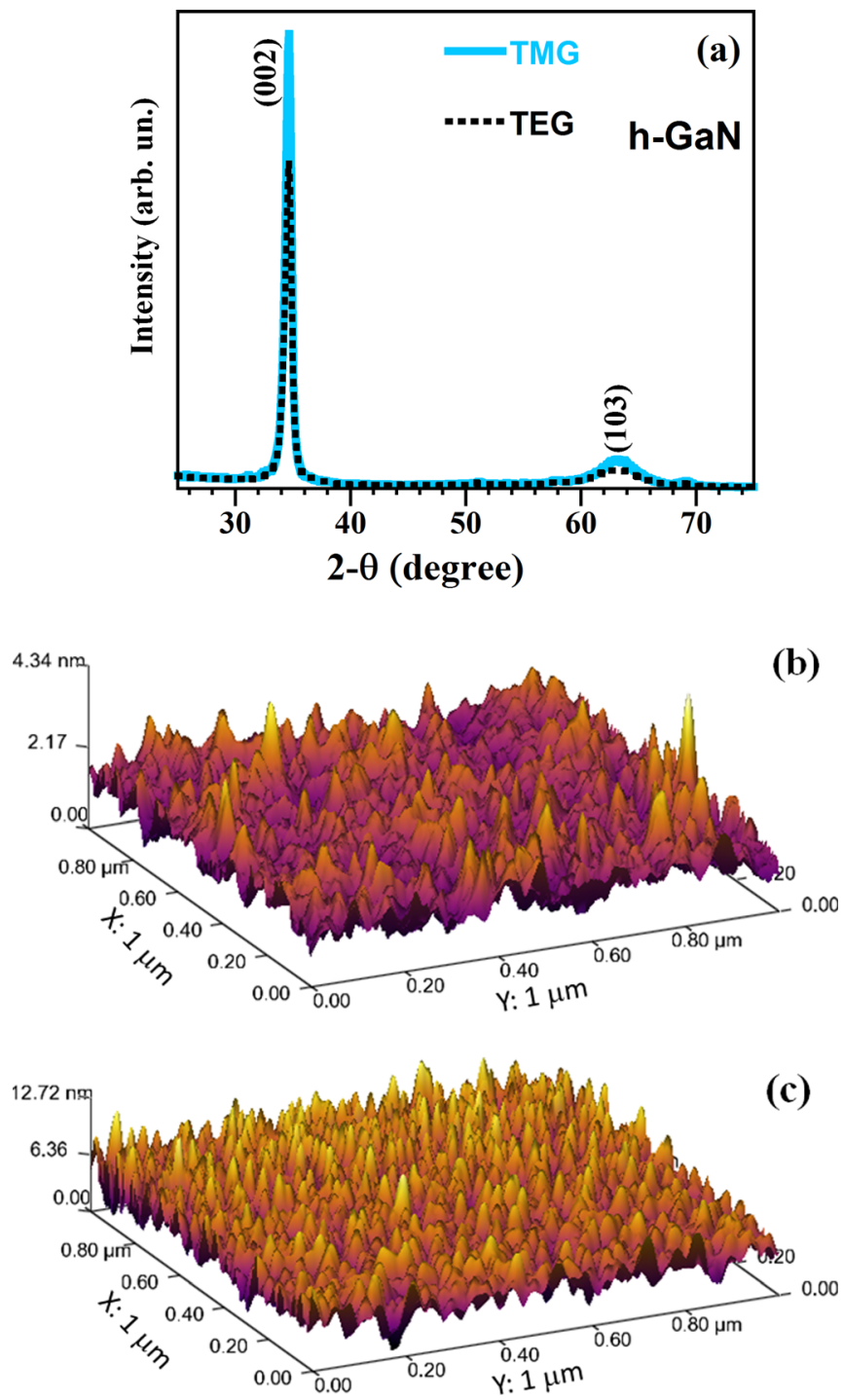

FIG. 2. (Color online) (a) GIXRD patterns of GaN thin films grown with TMG and TEG on Si (100) substrates at $200^{\circ}$ C. Surface morphologies of GaN films grown using (b) TEG and (c) TMG.

TMG precursors promote the formation of larger grains which result in a rougher surface, as shown in Fig. 2(c). ${ }^{14}$

The Ga $3 d$ and N $1 s$ high resolution XPS (HR-XPS) for the samples grown with TMG [(a) and (b)] and TEG [(c) and (d)] are displayed in Fig. 3 and obtained from the bulk parts of GaN films. Ga $3 d$ spectrum for TMG-grown GaN film is decomposed into two components at 19.28 and $18.17 \mathrm{eV}$, which can be assigned to Ga-N and Ga-Ga bonding states, respectively. ${ }^{11,15} \mathrm{~N} 1 s$ spectrum for TMG-grown GaN film is also decomposed into two components at 396.37 and $394.57 \mathrm{eV}$, which is assigned to the N-Ga bond and Auger Ga peaks. The spectral locations and intensities of Ga $3 d$ and $\mathrm{N} 1 s$ peaks were found to be slightly different for TEGgrown GaN sample. The Ga $3 d$ HR-XPS obtained for TEGgrown GaN film was fitted by two subpeaks located at 19.00 and $17.85 \mathrm{eV}$, corresponding to $\mathrm{Ga}-\mathrm{N}$ and $\mathrm{Ga}-\mathrm{Ga}$ bonds, respectively. The $\mathrm{N} 1 s$ scan obtained from TEG-grown GaN was fitted similarly using two subpeaks located at 396.55 and $394.75 \mathrm{eV}$, corresponding to N-Ga and Auger Ga bonds, respectively. Ga $3 d$ and $\mathrm{N} 1 s$ spectra obtained from $\mathrm{GaN}$ films grown with both TMG and TEG were in good agreement with the epitaxial grown GaN samples reported in the literature. ${ }^{11}$ No carbon signals were observed by the XPS measurement, indicating that ethyl groups in TEG and methyl groups in TMG were efficiently removed during the exposure periods of $\mathrm{N}_{2} / \mathrm{H}_{2}$ plasma. The $\mathrm{Ga}$ and $\mathrm{N}$ elemental compositions of GaN films were determined using XPS, where 33.4 at. $\% \mathrm{Ga}$ and 63.42 at. $\% \mathrm{~N}$ for TEG grown GaN and 30.49 at. $\% \mathrm{Ga}$ and 65.32 at. \% for TMG grown GaN are reported. Even though these results indicate that both films are nitrogen rich, it should be noted that the atomic concentration of $\mathrm{N}$ is overestimated due to the significant contribution of Auger Ga peaks, which overlap with the N 1s peak. Some gallium might have been lost between source and growth area during the growth and require further optimization of $\mathrm{N}_{2} / \mathrm{H}_{2}$ (nitrogen to hydrogen) ratio. The change in metalorganic precursor did not significantly affect the chemical compositions of the grown films.

Figure 4 shows O $1 s$ XPS spectra for the GaN samples grown with TMG (a) and TEG (b) and obtained from the bulk films $\left(t_{\mathrm{etch}}=200\right.$ and $150 \mathrm{~s}$ for TMG and TEG grown $\mathrm{GaN}$, respectively). The $\mathrm{O} 1 \mathrm{~s}$ spectra were fitted with a single peak located at $530.35 \mathrm{eV}$ for TMG grown GaN and at $531.6 \mathrm{eV}$ for TEG grown GaN. The $531.6 \mathrm{eV}$ peak can be assigned to chemisorbed oxygen atom and the $530.35 \mathrm{eV}$ is also due to the chemisorbed oxygen atom and difference in the binding energy is attributed to the difference in the chemical states. ${ }^{15}$ These oxygen species originate mainly from the atmospheric contamination. The atomic compositions of $\mathrm{O}$ are 3.89 at. \% for TMG-grown and 3.18 at. \% for TEG-grown GaN samples. It can be noted that the oxygen composition slightly increases for TMG grown GaN. This might be explained with the rough layer obtained from the spectroscopic ellipsometer analysis and high roughness obtained from the AFM analysis.

The refractive index $(n)$, extinction coefficient $(k)$, and optical band edge values $\left(E_{\mathrm{g}}\right)$ were determined from spectroscopic ellipsometry measurements, which are depicted in Fig. 5. In order to model the spectroscopic ellipsometry spectra, we followed two steps: Cauchy dispersion function was used for transparent wavelength region as the first step. In the following step, by using the optical constants obtained from the first step, Tauc-Lorentz model was used for the absorbing wavelength region of GaN films. The measured ellipsometry data of TMG grown GaN film were fitted by assuming two layer structures with a bulklike GaN layer on the Si substrate and a top surface roughness layer. The surface roughness layer is modeled as a mixture of bulk GaN material and voids. The fitting results provide thickness values for TMG-grown GaN layer as the following: $56.88 \mathrm{~nm}$ for $\mathrm{GaN}$ bulk layer and $11.22 \mathrm{~nm}$ for rough $\mathrm{GaN}$ layer. On the other hand, the measured ellipsometry data for TEGgrown GaN film was fitted by assuming only GaN bulk layer and the thickness of $\mathrm{GaN}$ layer is found to be $49 \mathrm{~nm}$. The value of refractive index at $632 \mathrm{~nm}$ is extracted as 2.31 and 2.18 for TMG- and TEG-grown GaN samples, respectively. GaN grown with TMG exhibits a significantly higher value 

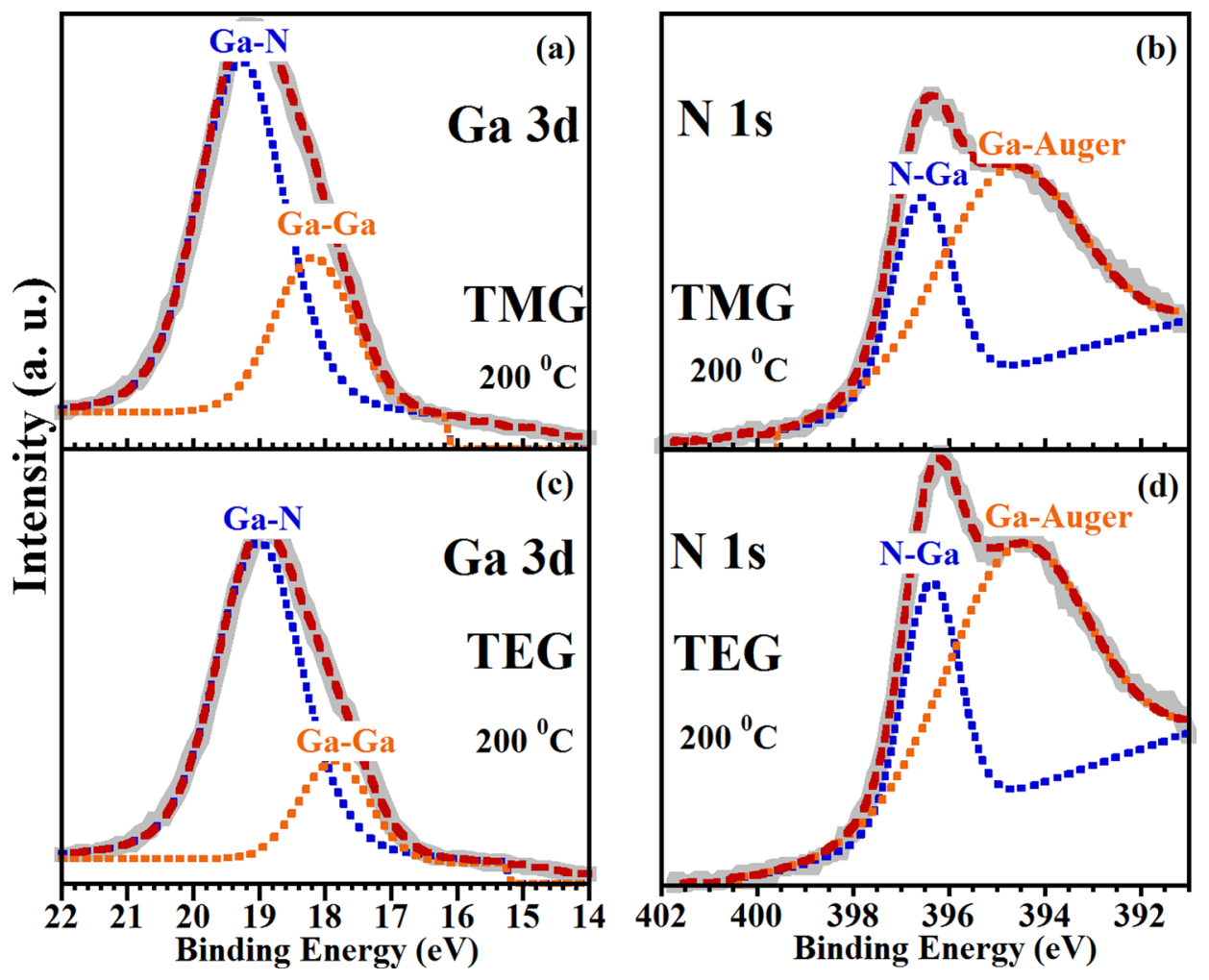

Fig. 3. (Color online) XPS spectra of (a) Ga $3 d$ and (b) N $1 s$ for the TMG grown GaN film and (c) Ga $3 d$ and (d) N $1 s$ for the TEG grown GaN film.

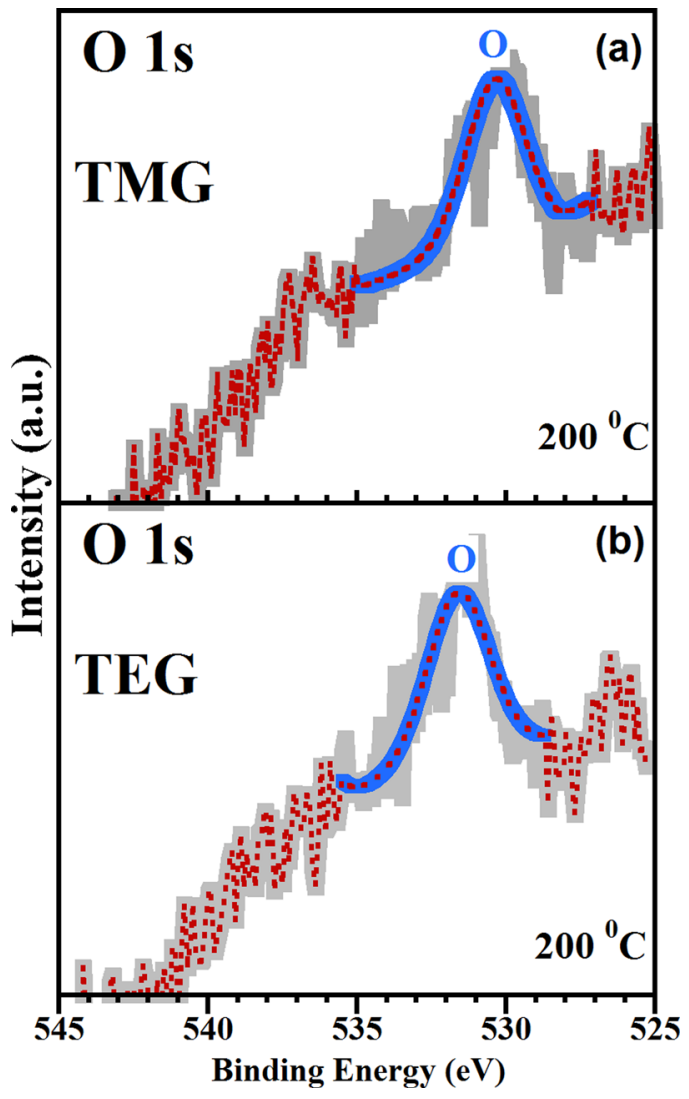

FIG. 4. (Color online) XPS spectra of O $1 s$ for (a) TMG-grown GaN film and (b) TEG-grown GaN film. of refractive index, which is pretty close to the hightemperature epitaxial-grown GaN counterparts. ${ }^{16}$ The refractive indices obtained for both $\mathrm{GaN}$ films are consistent with the refractive index values of the reported polycrystalline $\mathrm{GaN}$ films. However, it is quite interesting to observe a rather drastic change in the refractive index values occurs with the use of TMG precursor. One can conclude that larger grain size and improved crystallinity, as manifested by XRD and AFM, leads to a higher refractive index. ${ }^{12}$

The absorption coefficient, $\alpha(\lambda)=4 \pi \mathrm{k}(\lambda) / \lambda$, was calculated from the $\mathrm{k}(\lambda)$ values determined from spectroscopic ellipsometry. Approximate optical band gap values were obtained via extrapolation of the linear part of the absorption spectrum to $(\alpha \mathrm{E})^{2}=0$. The band gap values obtained for TMG- and TEG-grown GaN are found to be 3.57 and $3.6 \mathrm{eV}$, respectively. The two numbers are both higher than the value of $3.43 \mathrm{eV}$ for bulk single crystalline $\mathrm{GaN}$, which might be due to the polycrystallinity and presence of crystallites with different orientations. ${ }^{16}$ The decrease in the optical band gap energy with the use of TMG might be an indication of improved crystallinity and is consistent with the GIXRD, AFM, and spectroscopic ellipsometry results.

To further gain insight about the film crystallinity and chemical bond structure, FTIR spectrometry measurements were carried out for the GaN films grown on $\mathrm{Si}(100)$ and are presented in Fig. 6. Here, we particularly focus on the Reststrahlen band between 450 and $700 \mathrm{~cm}^{-1}$, which is centered about $570 \mathrm{~cm}^{-1}$ since no additional information was obtained in the high frequency range. The experimental IR spectra for both GaN samples agree well with the calculated 

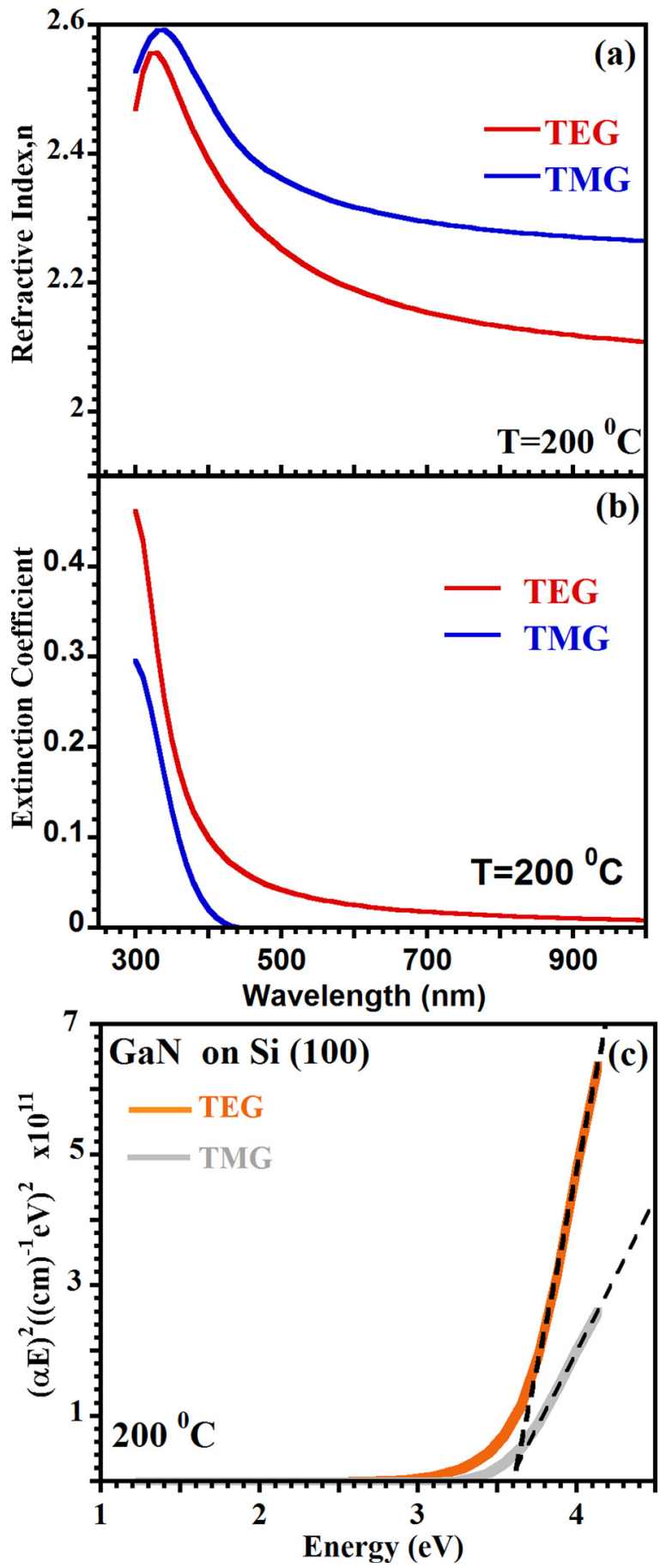

FIG. 5. (Color online) Dispersion of (a) refractive index and (b) extinction coefficient with wavelength extracted from ellipsometric data for TMG and TEG grown GaN films. (c) Optical band edge obtained from the spectroscopic ellipsometry measurement.

Reststrahlen band for a single crystalline wurtzite GaN film grown on $\mathrm{Si}$ (100). ${ }^{17}$ There is no significant discrepancy between the spectra of GaN samples grown with both precursors. The reflectance intensity of Reststrahlen band increases with the use of TMG precursor, which again confirms the improved crystallinity. ${ }^{17}$ The main peak at $\sim 570 \mathrm{~cm}^{-1}$ is due to the reflection from the $E_{1}(\mathrm{TO})$ phonon mode of wurtzite $\mathrm{GaN}$ and $E_{1}(\mathrm{TO})$ can be obtained through fitting $\left(555 \mathrm{~cm}^{-1}\right)$. These assignments are in good agreement

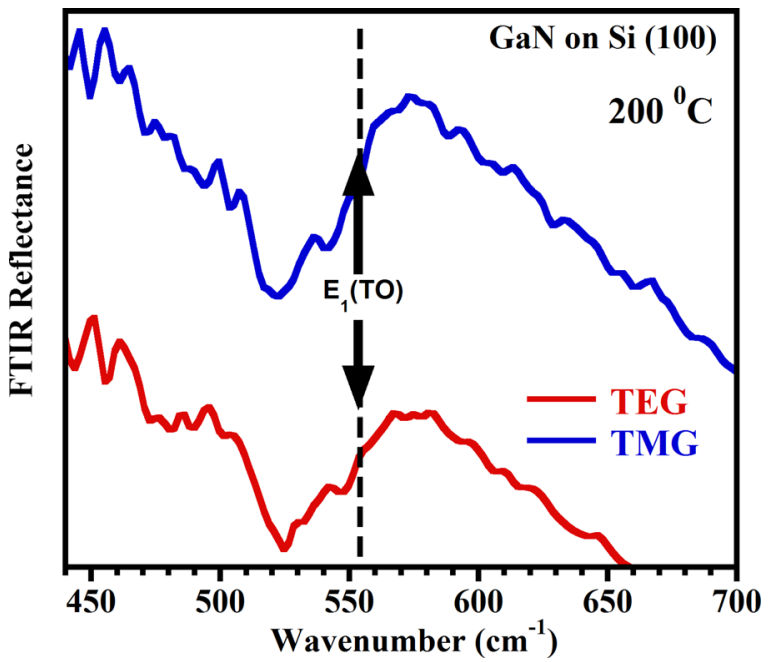

FIG. 6. (Color online) Experimental IR reflectance spectra for TMG and TEG grown GaN films.

with the values reported for $E_{1}(\mathrm{TO})$ at $557 \mathrm{~cm}^{-1}$ in the literature. $^{17}$

\section{CONCLUSIONS}

In summary, selection of group-III metal precursor has a significant effect on the crystalline quality and optical properties of the low-temperature hollow-cathode PA-ALD grown GaN films. We conclude that TMG might be a better gallium source for atomic layer deposition at low substrate temperatures $\left(<300{ }^{\circ} \mathrm{C}\right)$ in contrast to superior results obtained for MOCVD-grown GaN using TEG precursor. ${ }^{18}$ The combination of the spectroscopic ellipsometry and AFM results strongly implies that the growth mechanism exhibits variations for different metallic precursors used during the PA-ALD growth of GaN films. The use of TMG precursor leads to an increase in both the crystallite size and mean grain size. The increase in the refractive index value with the use TMG precursor is consistent with larger crystallite and grain sizes. The FTIR spectrum identified the wurtzite crystalline structure nature of the grown GaN films with both precursors. Higher reflectance in Reststrahlen band indicates decent crystalline quality for TMG grown GaN films. On the other hand, experimental results presented in this study confirm that TMG-grown GaN films exhibit surfaces with higher roughness values.

\section{ACKNOWLEDGMENTS}

M.A. acknowledges the financial support from TUBITAK Grant No. 114F002 and Marmara University BAPKO Project Nos. FEN-A-110913-0378 and FEN-D-1305150201. N.B. acknowledges financial support from TUBITAK Grant Nos. 112M482 and 214M015. A.H. and S.A.L. acknowledge Higher Education Commission of Pakistan (HEC) for Human resource development (HRD) fellowship for M.S. leading to Ph.D.

${ }^{1}$ K. M. A. Saron, M. R. Hashim, and M. A. Farrukh, Superlattices Microstruct. 64, 88 (2013). 
${ }^{2}$ F. Medjdoub, M. Zegaoui, B. Grimbert, D. Ducatteau, N. Rolland, and P. A. Rolland, IEEE Electron Device Lett. 33, 1168 (2012).

${ }^{3}$ D. Zhu, D. J. Wallis, and C. J. Humphreys, Rep. Prog. Phys. 76, 106501 (2013).

${ }^{4}$ S. Chowdhury, Phys. Status Solidi A 212, 1066 (2015).

${ }^{5}$ G. Li, W. Wang, W. Yang, and H. Wang, Surf. Sci. Rep. 70, 380 (2015).

${ }^{6}$ P. Motamedi, N. Dalili, and K. Cadien, J. Mater. Chem. C 3, 7428 (2015).

${ }^{7}$ K. Shen, M. Jiang, H. Liu, H. Hsueh, Y. Kao, R. Horng, and D. Wuu, Opt. Express 21, 26468 (2013).

${ }^{8}$ M. Junaid, C. Hsiao, J. Palisaitis, J. Jensen, P. O. Persson, L. Hultman, and J. Birch, Appl. Phys. Lett. 98, 141915 (2011).

${ }^{9}$ C. Ozgit, I. Donmez, M. Alevli, and N. Biyikli, J. Vac. Sci. Technol. A 30, 01A124 (2012)

${ }^{10}$ C. Ozgit-Akgun, F. Kayaci, S. Vempati, A. Haider, A. Celebioglu, E. Goldenberg, S. Kizir, T. Uyar, and N. Biyikli, J. Mater. Chem. C 3, 5199 (2015).
${ }^{11}$ C. Ozgit-Akgun, E. Goldenberg, A. K. Okyay, and N. Biyikli, J. Mater. Chem. C 2, 2123 (2014); and references therein.

${ }^{12}$ P. Motamedi and K. Cadien, RSC Adv. 5, 57865 (2015).

${ }^{13}$ H. Y. Shih, M. C. Lin, L. Y. Chen, and M. J. Chen, Nanotechnology 26, 014002 (2015).

${ }^{14}$ H. Van Bui, F. B. Wiggers, A. Gupta, M. D. Nguyen, A. A. I. Aarnink, M. P. de Jong, and A. Y. Kovalgin, J. Vac. Sci. Technol. A. 33, 01A111 (2015).

${ }^{15}$ D. Li, M. Sumiya, S. Fuke, D. Yang, D. Que, Y. Suzuki, and Y. Fukuda, J. Appl. Phys. 90, 4219 (2001).

${ }^{16}$ A. Biswas, D. Bahttaccharyya, N. K. Sahoo, B. S. Yadav, S. S. Major, and R. S. Srinivasa, J. Appl. Phys. 103, 083541 (2008).

${ }^{17}$ X. Zhang, Y. T. Hou, Z. C. Feng, and J. L. Chen, J. Appl. Phys. 89, 6165 (2001).

${ }^{18}$ A. Saxler, D. Walker, P. Kung, X. Zhang, M. Razeghi, J. Solomon, W. Mitchel, and H. R. Vydyanath, Appl. Phys. Lett. 71, 3272 (1997). 